\title{
“HERITAGE \& DEVELOPMENT” STRATEGY ON HISTORIC URBAN LANDSCAPE (HUL): THE ADDED VALUE OF MULTI-TEMPORAL HUB APPLICATION.
}

\author{
M. Yang a , R. Brumana ${ }^{\text {b }}$, M. Previtali ${ }^{b *}$

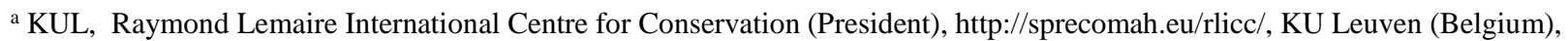 \\ Kasteelpark Arenberg 1 B-3001 Leuven (Heverlee) - (minja.yang@gmail.com); \\ b Politecnico di Milano, Department of Department of Architecture, Built Environment and Construction Engineering, DABC LAB \\ GIcarus, Via Ponzio 31, 20133 - (raffaella.brumana)@mail.polimi.it;
}

KEY WORDS: Map Heritage, Landscape, Open Data, Virtual Hub, Planning, GEODB Hub, HUL

\begin{abstract}
:
Growing interest in boosting urban identity and character is increasing the demand for historic maps and documents of cities in constant evolution as in the case of metropolitan areas, peripheries and riverbank sites. A 'Heritage \& Development strategy based on the Historic Urban Landscape approach is required by city makers to ensure that exploitation needs can valorise the site's natural and cultural heritage for sustainable social and economic optimisation.

This paper intends to propose innovative virtual hub technologies of brokering, discovering and accessing open data, making available - to a large public of users - the multi-temporal dense stratified evidences of the targeted built environment areas and its surroundings, through the navigation of geo-referenced historical maps, together with current maps, going through design data. Enhancing the practice of publishing maps as open data represents a powerful leverage for time and cost effectiveness among planners, scientists and historians; soliciting their use to gain a vast knowledge of that areas, including a better comprehension of the transformations over the centuries, in order to support decision-making analysis, environmental monitoring and urban area planning; thus contribute to improving the sustainability of interventions respectful of the identity of the urban landscape. A case study of the 'Deux Rives' planning area in the city of Strasbourg is presented to illustrate these issues. The City of Strasbourg's commendable work in publishing the historical maps supports the municipal authority's Heritage \& Development strategy in meeting contemporary and future needs while mitigating long-term damage from pressures for new interventions. Innovative virtual hub based applications to gather open data coming from different sources (city territorial services, design data sources, mobility services) can result in immeasurable added value through communicating the wealth of the planned areas and raising awareness among citizens and visitors of the interventions.
\end{abstract}

\section{INTRODUCTION}

Growing interest in urban identity, character and the historic urban landscape approach by city makers, including elected and technical officers, real estate developers and of course the inhabitants, have increased demand for historic documents of cities undergoing constant evolution. The proactive role played by Cities and Local Governments through various forms of public and private partnership have been presented in numerous reports of Agenda 21 for Culture, contributing progressively to replication and adaptation in many countries (Yang, 2014).

Growing availability of data on the Historic Urban Landscape (HUL) domain at the territorial and urban landscape scale will improve analysis among planners, notably in addressing preservation policies at a larger scale to make heritage a vector for territorial development, rather than for heritage to be confined into a limited conservation district. Through dissemination of best practice cases that link city governance to urban planning, mobility, tourism, economic development and quality of life in general, awareness by the citizens and tourists of the rich history and wealth bequeathed from the past can lead to better appraisal of new intervention needs and engender participatory and democratic city-making among stakeholders.

Digital historic cartography can serve to promote the humanistic component of city and territorial development by enabling historians, scientists, architects, planners, and technical engineers among other professionals, as well as the general inhabitants and the commercial-business sectors in finding a common vision to face the territorial challenges of a rapidly urbanising world. With $70 \%$ of the EU population living in urban agglomerations, cities have undergone important changes over the past two centuries, notably in the urban peripheries where industrialisation have fragmented agricultural land, often leaving behind disconnected urban areas or desolate postindustrial peri-urban zones. Greater awareness of the modification over the centuries in natural features such as waterways, through artificial canalisation, riverbeds or wetland registered in historical maps over the last centuries, can serve in valorising the territory for new design projects and development plans.

With regard to both the municipality scale, and the territorial scale of landscapes, a semantic based application has been implemented to facilitate the navigation across time and space through multi-temporal and multi-scale geospatial open data access based on the policy context.

A considerable number of Directives and Conventions in Europe that concerns geospatial information and also specific features of the landscape, create a complex and dynamic policy context: to name a few, INSPIRE Directive on the great value of Open Data publishing and sharing, Common Agriculture Policy (CAP), meet Habitat Directive (Natura2000), European Landscape Convention (Florence Convention), European Convention on the Protection of the Archaeological Heritage (Valetta Convention) and World Heritage Convention (in its framework for Cultural Landscapes).

Cross sectorial analyses enabled by technical opportunities of Open Data sharing can forge HUL objectives in both strategy development and interventions. 


\section{2. "HERITAGE \& DEVELOPMENT" STRATEGY ON HISTORIC URBAN LANDSCAPE (HUL) APPROACH}

The Historic Urban Landscape (HUL) approach is holistic and interdisciplinary aimed to address the inclusive management of heritage resources in dynamic and constantly evolving environments, in both the historic districts of cities and their surrounding territories. As noted in the Recommendation adopted by UNESCO in 2011, "the historic urban landscape is the urban area understood as the result of a historic layering of cultural and natural values and attributes, extending beyond the notion of 'historic centre' or 'ensemble' to include the broader urban context and its geographical setting. This wider context includes notably the site's topography, geomorphology, hydrology and natural features, its built environment, both historic and contemporary, its infrastructures above and below ground, its open spaces and gardens, its land use patterns and spatial organisation, perceptions and visual relationships, as well as all other elements of the urban structure. It also includes social and cultural practices and values, economic processes and the intangible dimensions of heritage as related to diversity and identity' (UNESCO, 2011).

The Six Critical Steps, outlined in the HUL Recommendation quoted in the HUL Guidebook (WHITRAP, 2016):

1. Undertake comprehensive surveys and mapping of the city's natural, cultural and human resources;

2. Reach consensus using participatory planning and stakeholder consultations on values to protect for transmission [...] and to determine the attributes that carry these values;

3. Assess vulnerability of these attributes to socioeconomic stresses and impacts of climate change;

4. Integrate urban heritage values and their vulnerability status into a wider framework of city development [...];

5. Prioritise actions for conservation and development;

6. Establish appropriate partnerships and local management frameworks for each of the identifies projects for conservation and development, as well as to develop mechanisms for the coordination of various activities between different actors, both public and private.

\section{3. “CASE STUDY: NEW URBAN DEVELOPMENT IN STRASBOURG - THE 'DEUX RIVES’ PROGRAMME}

This case presents the implementation of the HUL approach in the 250 ha new urban development in Strasbourg, called the Deux Rives. This interesting project of turning a former industrial riverport into a new mixed-use area aims to promote Eurodistrict Strasbourg-Ortenau, the franco-german crossborder entity located adjacent to the World Heritage listed Grande Ile-Neustadt of Strasbourg, recognized for its outstanding universal value depicting the franco-german shared heritage.

The overall urban plan has been developed by Henri Bava of TER, a landscape design company, winner of the prestigious 2018 Grand Prix d'Urbanism of France; particular attention is being given for the new urban extension to be linked to both its cultural and natural heritage. The core area of this former industrial zone called Coop (area of cooperative warehouses) is being turned into a cultural centre including a conversion of a building into a storage depot of art objects of the 11 museums owned and managed by the City of Strasbourg. Architect Alexandre Chemetoff is preparing the design of this area. An inventory of the industrial heritage of the Deux Rives has been carried out with the involvement of RLICC faculty and students to enable a better understanding of the complexity of an area rich in history and dense with future economic and social opportunities.

\subsection{Historic cities, maps digitization and publication made on the city of Strasburg}

The Department of Urbanism of Strasbourg headed by Eric Chenderowsky, a heritage sensitive planner has been working for many years on the digitized map of the city from its Roman origin to the present. Its Geomatic \& Territorial Knowledge Service (Service Géomatique et Connaissance du Territoire) headed by Olivier Banaszak have already georeferenced and published many historical maps belonging to the different periods of the city (Figure 1).

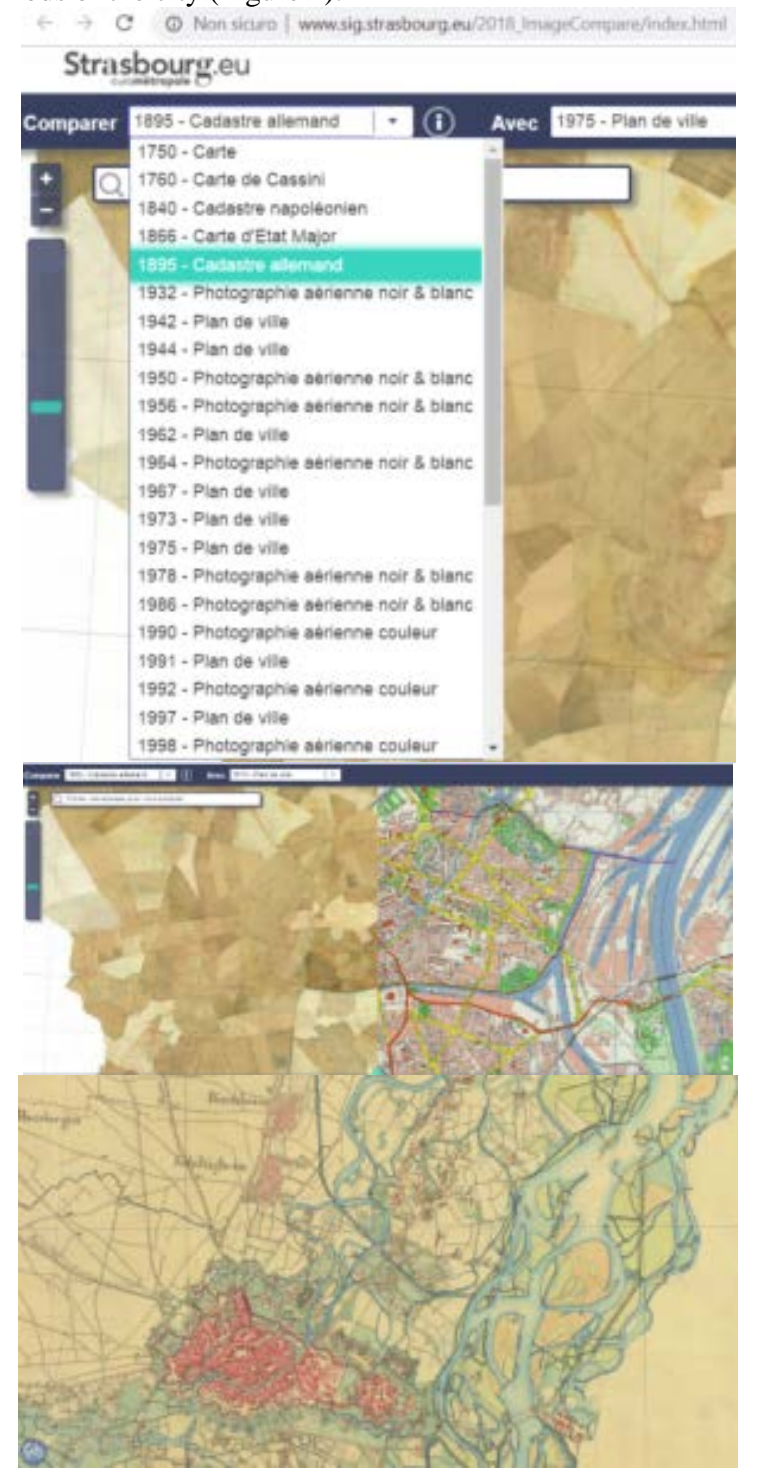

Figure 1. "Strasbourg au fil du temps": the website implemented by the City of Strasbourg with the list historical georeferenced maps (upper). The cadastre allemande (1895) coupled to the current map of the city (centre). Below a detail of the French map (Carte de l'Etat-Major 1820-1866), the Strasbourg area before the design of the German administration (1871-1918) 
Here are two links to the resources published:

- $\quad$ historical maps published in pdf format: http://sig.strasbourg.eu/index.php?page=cartotheque;

- $\quad$ "Strasbourg over time" « Strasbourg au fil du temps », a tool developed by the Service to allow the overlay and comparison of historical maps and historical data across the centuries along the Deux Rives development area (Figure 1): http://www.sig.strasbourg.eu/2018_ImageCompare/in dex.html.

The historical map data published on the internet are to date only the face of the larger stock of scans of historical plans collected over the years. All the maps can be selected and compared to each other. A number of tools allowing different degrees of transparency, to superimpose or to couple the maps are available to the users.

\subsection{The design project of the Deux Rives: Heritage \& Development strategy on HUL}

Under the political leadership of the Mayor of Strasbourg, Roland Ries, a former professor of French literature, committed to building a human-centred city, the "heritage \& development" strategy is slowly but surely taking shape. His ambitious electoral commitment of increasing the housing stock by 3000 units a year, of which 30 percent in social housing and "first acquisition home", the real estate development investors are under regulatory obligations to respect the HUL approach for projects in both old and new buildings and sites. Calling upon the private sector to respect heritage and sound ecological practices, this new urban area is already registered by the French Government as one of the innovative ecological projects of France in its quest to meet Goal 11 of the 2030 SDGs as well as the New Urban Agenda of UN Habitat. UNESCO maintains a careful watch over the city as one of the HUL examples in action, promoting buildings in thermic-proven brick in respect of ecology and in memory of the industrial heritage, providing opportunities for contemporary architectural expressions and creativity while in visual harmony with the World Heritage area of Strasbourg.

The numerous historic maps of this city that changed "nationality" between France \& Germany several times attest the role that innovation and excellence have resulted from a competition between nations while creating its own singularity in the culture of shared heritage.

The WH Site of the Grande-Île and Neustadt in Strasbourg, initially inscribed in 1988 for the Grande-Île, the historic centre of Strasbourg, structured around the cathedral was extended in 2018 to include Neustadt, the new town, designed and built under the German administration (1871-1918).

Drawing its urban inspiration partially from the model of Haussmann, who carried out a massive urban renewal programme under Emperor Napoleon III, while adopting an architectural idiom of Germanic inspiration, this dual influence has enabled the creation of an urban space that is specific to Strasbourg, where the perspectives created around the cathedral open up to a unified landscape of rivers and canals.

During the construction of the tramway in the 1990s, there was a major "preventive archaeological" research in the city centre resulting in a wealth of historic information. For the new extension area of 250 ha including the 74 ha of the latest ZAC (special zone), there is a need for a more thorough documentation \& recording work, needed to understand its industrial heritage in its relationship with the city and the surrounding region.
The Deux Rives Eco-city is a key feature of the overall project and is designed to provide an inventive, crosscutting approach to the sustainable city in the area of the intervention. Initiatives in the area covered by SPL Deux-Rives (Deux Rives concerted development zone) include sustainable mobility, the "activation guide-plan" and its actions relating to culture and citizens' involvement, energy performance and supply strategy, ground remediation and the development of the Coop project as illustrated by the design project (Figure 2).

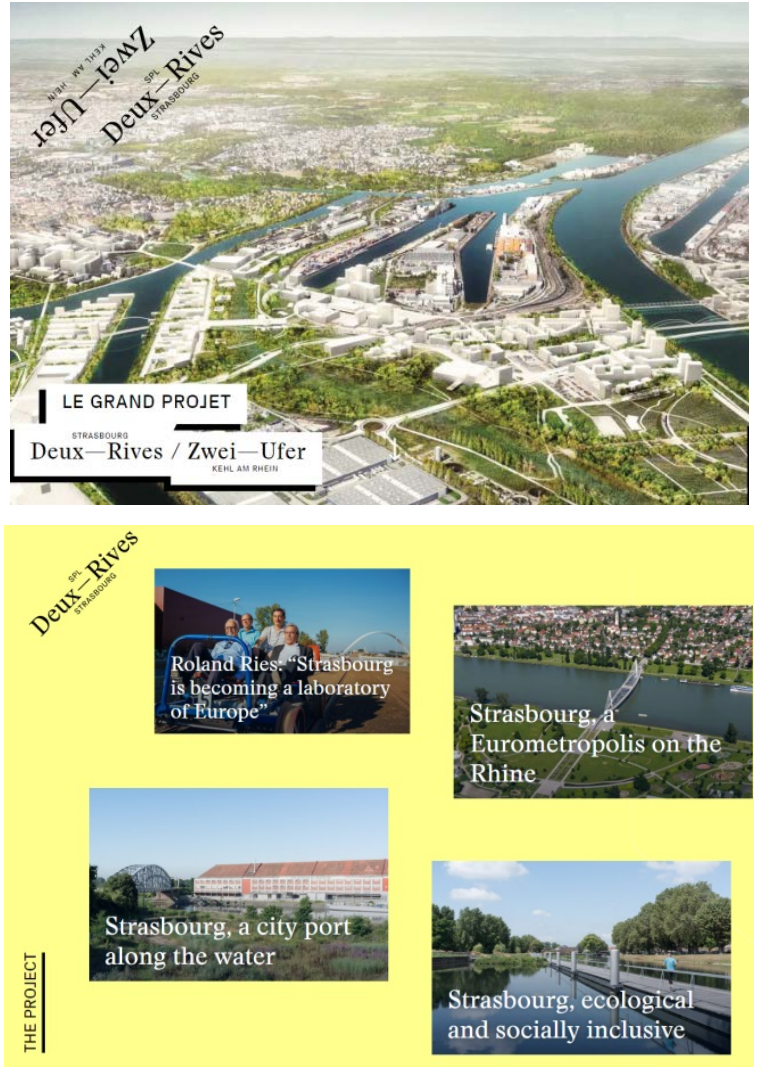

Figure 2. An overview of the Deux Rives Project and details (https://strasbourgdeuxrives.eu/en/).

The Eco-city project aims to be "a driving force for social and environmental innovation, including the 'Pass Mobilité', shared parking, community housing, wood constructions, positive energy tower block, cultural urban-project and citizen activation". SPL Deux-Rives has also introduced a "sustainable development and environment reference document for the Deux-Rives”, based on the Strasbourg Eurometropolis reference document.

\section{SEMANTIC BASED VIRTUAL HUBS APPLICATION AS AN ADDED VALUE TO LEVERAGING OPEN DATA DISTRIBUTION, DISCOVERY AND ACCESS.}

Map data collection and publication technology are demonstrating that they can be useful for different analysis on the historic layers of a city for planning, built environment and transformation (Cuca at al., 2013) and also for the inhabitants to understand their city (Brumana et al., 2018a) through not only the built heritage but also by juxtaposing the "heritage" area with socio-economic data of the inhabitants over several decades. Open access to historical information for landscape analysis with the help of Spatial Data Infrastructure have been progressively applied (Brumana et al, 2013; Brumana et al., 2015). The growth of geo-portals as the one shown of the city of 
Strasbourg proves that great efforts have been made in this direction over the last decade.

\subsection{Virtual Hubs}

What could be said is that there are many data sources publishing data in different levels of open data and formats, but with scarce connection to one other. This limits the potential in interconnection. Innovative technologies like Virtual Hubs have been introduced in the domain of large big data sets: that is the case of the Virtual Hub set up to Discovery and Access Brokering service to support Global Earth Observation (GEO$\mathrm{DAB})$, requiring integration with other data sources related to various purposes: climate change, health, water, air pollution, and others (Nativi et al., 2013).

Virtual Hubs are in constant development, to respond to the demand of improving instruments capable of discovering and accessing available and published open data without duplicating the data source and for improving the data use for different purposes.

The GeoPan application (Figure 3) has been developed to start facing the granularity of many geospatial data sources with few data (as in the case of the local geo-portals, publishing historical maps and current maps, together with other data) but with a great dispersion in the territory, with thousands of cases without connections: GeoPan application was developed on top of a pan-European Virtual Hub implemented within the EU project ENERGIC OD (Mazzetti et al., 2015). The term virtual hub is adopted referring to the hub-spoke distribution paradigm. An ENERGIC OD Virtual Hub (VH) is defined as a virtual node where users can seamlessly access potentially unlimited datasets by brokering heterogeneous geospatial data sources. In particular, these statements underline some key aspects of the Virtual Hub concept:

- an ENERGIC OD Hub is virtual: this means that the data sources connected are not duplicated into a new infrastructure but are accessed in their original repository;

- an ENERGIC OD VH accesses datasets in a seamless way from heterogeneous geospatial data sources: the heterogeneity in data formats, standards and protocols is solved by the $\mathrm{VH}$, allowing a higher interoperability between services and datasets;

- an ENERGIC OD VH can access potentially unlimited datasets: the system is able to face the Big Data issue of data Volume allowing to access potentially unlimited datasets;

- an ENERGIC OD VH is based on a brokering architecture: a software architecture adopting the(message) broker pattern is usually referred as a hub-and-spoke architecture;

- an ENERGIC OD VH is aimed to connect heterogeneous geospatial data sources: different geospatial data sources can be connected by using a VH.

In particular, an ENERGIC OD VH is a solution able to reduce the interoperability problems between data providers and applications. It requires to extend the Service Oriented Architecture (SOA) archetype by introducing a new component that interconnects the different service protocols and standards, mediating their models and interface specifications. In addition, this component must work out all the necessary distribution and virtualisation capabilities to lower the entry barriers for multidisciplinary applications. Specifically, ENERGIC OD VH, relies upon a Brokering approach, with APP development for the generation of web applications based on historical maps (Previtali and Latre, 2018).

The Italian version of the application (Figure 3) was designed in close contact with the Lombardy Geologist association to track historical maps of riverbed changes and link them with flooding hydrogeological risk (Geopan PRO) and urban transformation (Geopan LIGHT). The experimentation of brokering based gateway to geo-referenced historical and current maps for risk analysis demonstrated that the integration of different data sets can improve the knowledge of the territory at a granular level, involving different communities, as in the case of the geologists merging different expertise and different data sources without any duplication (Previtali, 2017).

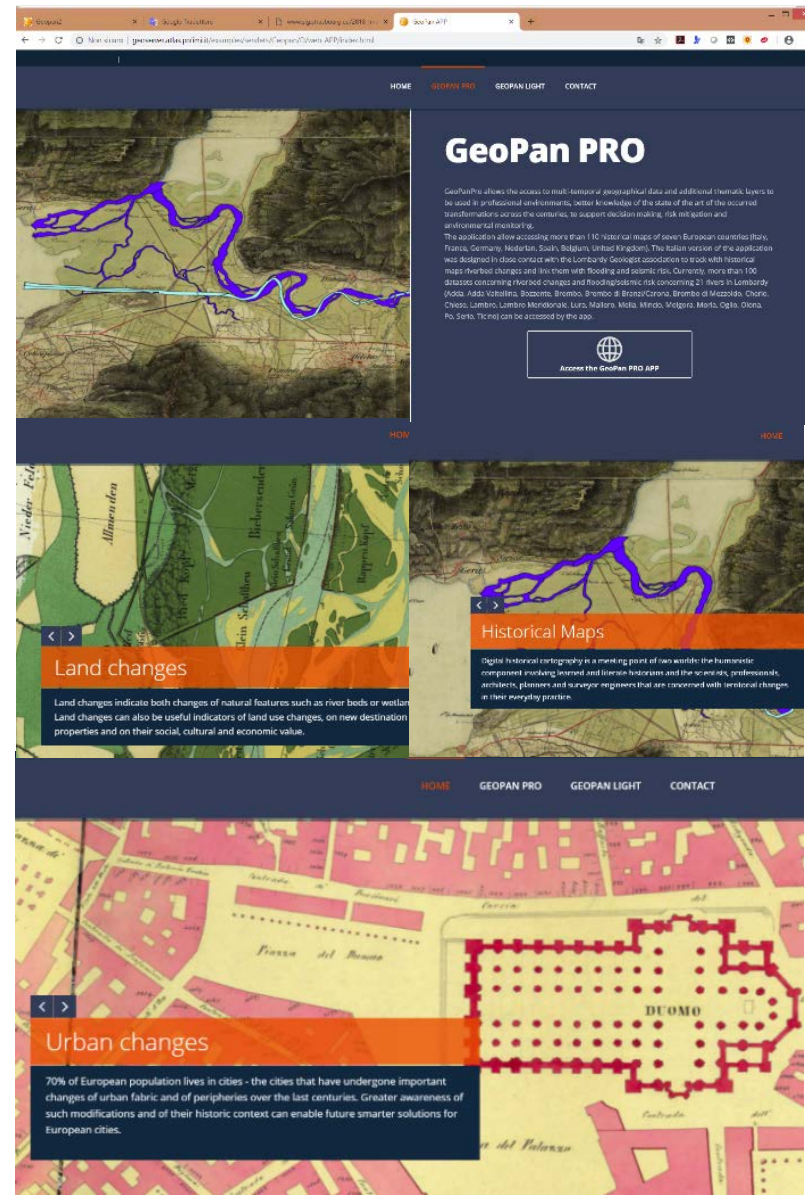

Figure 3. The GEOPAN APP: an application where to meet, discover and access Geographic Open Data based on Virtual Hub at the built environment domain (Lombardy Region) and urban domain (City of Milan).

A great advantage of the brokered approach, is that no common model is defined, and each system can adopt or maintain their preferred interfaces, metadata and data models. Specific components (the brokers) are in charge of accessing the participant systems, providing all the required mediation and harmonisation functionalities. The only interoperability agreement is the availability of documentation describing the published interfaces, metadata and data models. No (major) reengineering of existing systems is required for connecting different services or other data sources. The brokered approach allows communities-of-practice free from defining their own 
specifications, and mediating between different specifications. Mediation will happen at the lowest common level between specifications, but it is generally sufficient for most interdisciplinary applications. Obviously brokering is not magic, the complexity of interoperability is still there, but it is simply shifted from data/product users and providers towards the brokers. Data users and providers are set free of interoperability - i.e. they do not have to make their clients and server compliant with specifications anymore - but new components, the brokers, are in charge of handling all the complexity. This shift of complexity from clients/servers to brokers has two main advantages: (a) it implements the general engineering pattern called "separation-of-concerns": where there is a specific functionality (interoperability), there should be a specific responsible (broker), (b) a third tier between clients and servers can host added-value services (e.g. semantics, data transformations). A broker is a mediator which implements some services (i.e. discover, access, distribution, added value, etc.) to facilitate the interconnection between client and server components in a Client-Server architecture.

\subsection{Metadata enrichment and semantic based search supported by the Virtual Hubs}

As stressed before, territory and city are complex systems in continuous evolution and transformation. To have a thorough understanding of the dynamics that have affected them, the study of ancient maps and a comparison with the modern ones is crucial. So, to enhance usability of the historical data and to assure their interoperability with modern cartography, their accessibility through Virtual Hub becomes an essential requirement. Virtual Hub, in fact, ensures interoperability giving the users a single point of access with a standard protocol and interface.

In this contest, the ENRGIC OD Virtual Hub can be seen as a sort of a switch to Open Data published by different source. A brokering service that works as the flight ticket (or hotels reservation) search hubs, allowing to 'see' the different data source of the different companies.

For the integration, specific attention is paid on geographic coverage, metadata availability and their compliance to standard profiles. This aspect is particularly significant since it allows verifying the quality and usability of data. Metadata filling are crucial for the semantic search operated by the Virtual Hub. Indeed, this search is operated in the keyword and in the description field of the published data set. In the specific case presented in this paper, data sets connected with the "Deux Rives" project can be published with the keywords as "riverside", "landscape", or "HUL" (Heritage Urban Landscape). Other metadata fields include: names of the Rivers, or Cities, dates of constructions of the historical maps, scale of representation, authors, Geographic Reference system, etc.

\section{GEOPAN APP FUNCTIONALITIES AND THE STRABOURG DEUX RIVES}

GeoPAN Atla@s is an updatable customisable application that allows access to a set of multi-temporal geographical data and additional thematic layers to be used in professional environments, published as Open Data maps formats.

The application allows accessing more than 110 historical maps of seven European countries (Italy, France, Germany, Nederland, Spain, Belgium, United Kingdom) that have been crawled by advanced searcher. And it is nowadays ready to be updated by different open maps, historical data, and design plans as well (Figure 4).

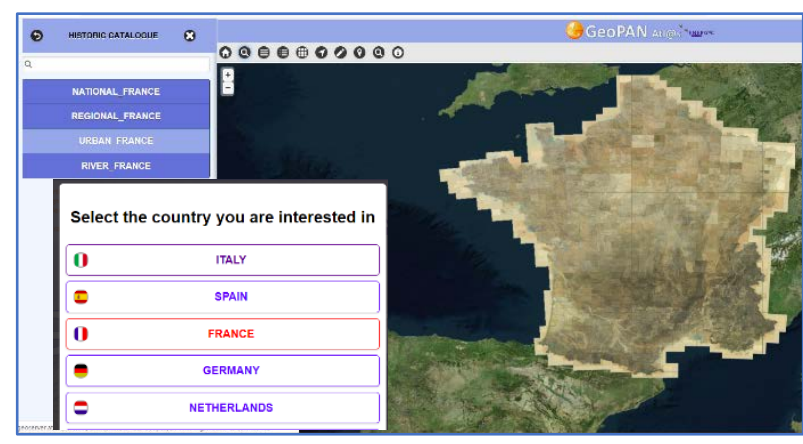

Figure 4. The GEOPAN APP search function and the crawled maps in French.

The application is ready to be updated by different data sources coming from websites publishing Open Data including historical and current maps, together with other Open Data (i.e. temperature, water level rising, raining parameters, pollutions).

The ground of this APP in terms of thematic fields of interests such as agriculture, biodiversity, ecosystems, urban planning, and so forth is addressed to support direct engagement of endusers communities such as planners, citizens, bikers, public and private bodies involved with landscape monitoring, protection, planning, management and fruition.

The GeoPan APP aims to facilitate by multiple users the access to different multi temporal data sets, coming from different sources (i.e. national archives as for the historical maps, satellite data, national and regional geo-portals, others): such data sets need to be discovered, accessed and geo-spatially related in a faster way by the users, once the datasets are made available following the Open Data protocols within the OGC framework.

The association of such data is often underemployed in research analysis and planning activities aiming to conjugate heritage and development matters, aiming to valorise the landscape content with traces of past transformation characterising the built environment as in the case here illustrated. Improvement of services related to the easy redistribution of Geographic Open Data shall tackle a massive adoption of the historical map analysis potential in support of the HUL analysis and Heritage \& Development Strategy. The case here described could be used as a vehicle contributing to spreading similar cases of pan European good practices in the context here described. Semantic search based on Heritage \& Development strategy and HUL words can represent an innovative entry point to boost the networking of similar experiences interested to join the platform to enhance the objectives of such approach.

The case study of Strasbourg represents the first experiment of updating and testing, as described in the following paragraph.

The main data sources integrated in this case are:

- geo-referenced maps published by the City of Strasburg ("Strasbourg au fil du temps") available as WMS services;

- maps of the Rhine river between Basel and Lauterburg (1830 - 1872) available as TMS and WMTS service published by the GICARUS server.

The maps of the Rhine river are quite important since the Upper Rhine from Basle downstream to the city of Worms was the most diversified part of the Rhine in the past and it is also known as the "furcation zone". Swift stretches extend over a length of about $190 \mathrm{~km}$. The canalisation of the Upper Rhine, 
the so-called Tulla rectification, was carried out between 1817 and 1876. Figure 5 a shows in red the design of the new riverbed and in blue the original one. The river was deepened and channelled between embankments, new sections were dug to straighten out its meandering course, and numerous small islands were removed. As a result, the length of the Rhine in this stretch had been shortened to $81 \mathrm{~km}$ (23 percent of total length). This is a very important change in the relationship between the city and the river that historical maps allows to track. The integration between the maps offered by the City of Strasbourg and the maps of the Tulla rectification provides important insights on the HUL of Strasbourg and its change over time.
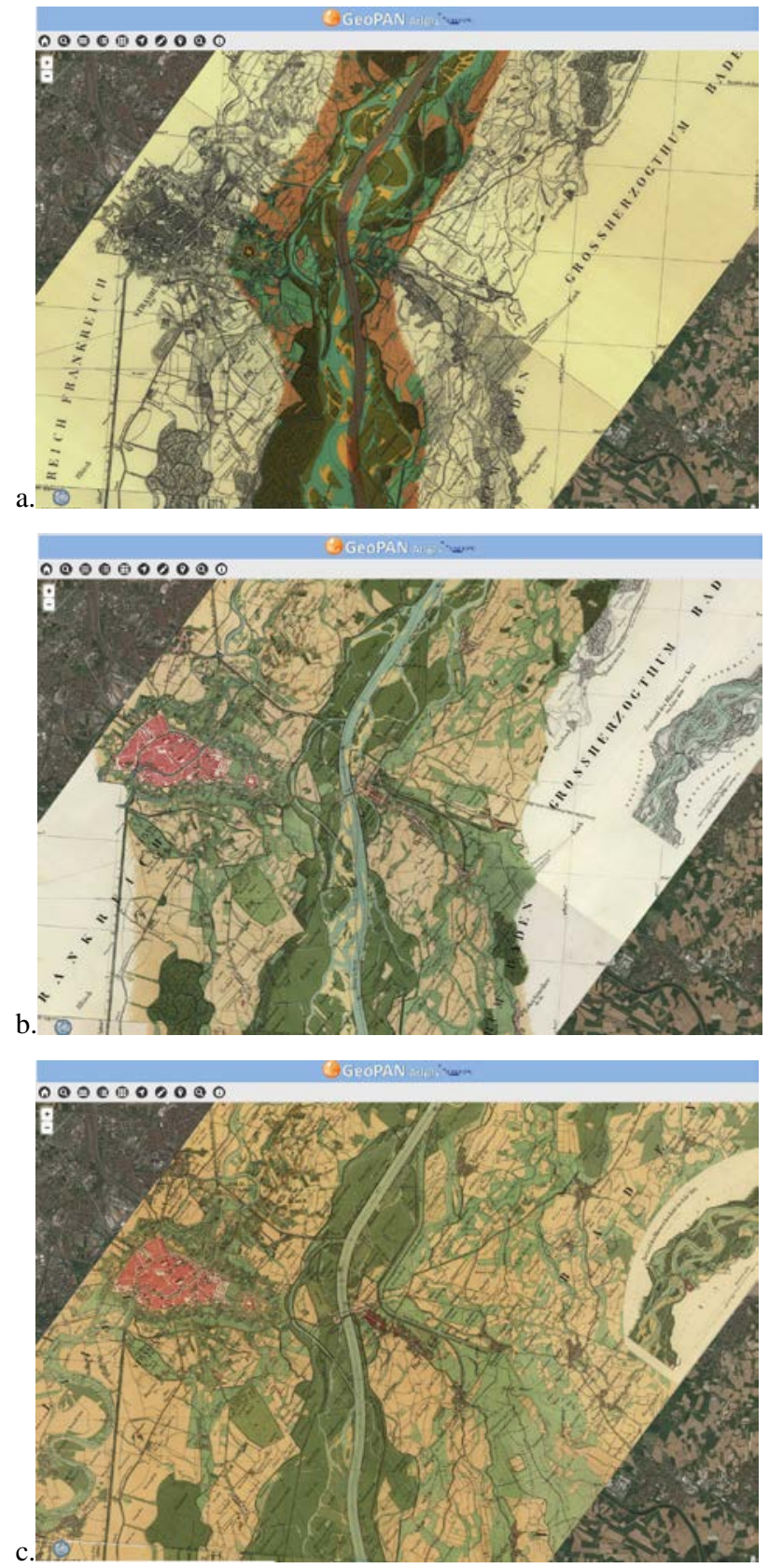

Figure 5. Rhine river in correspondence of Strasbourg: the original riverbed in 1830 (light blue) and the rectification project (red) (a), the riverbed (light blue) in 1852 (b) and 1872 (c).

Integration of such maps coming from different data sources was facilitated by the adoption of a Virtual Hub framework.
Indeed, integration is achieved without any duplication of the data source (Figure 6).

Once the maps data of the city of Strasbourg are retrieved, without any duplication of the data source, one of the functionalities of the GeoPan Atl@s regards "layer handling" (Figure 5) that allows to access and to turn "on" and "off" the layers of Open Geo Data obtained by a specific query (http://geoserver.atlas.polimi.it/examples/servlets/Geopan/D/Ent ry/selector2b.html).
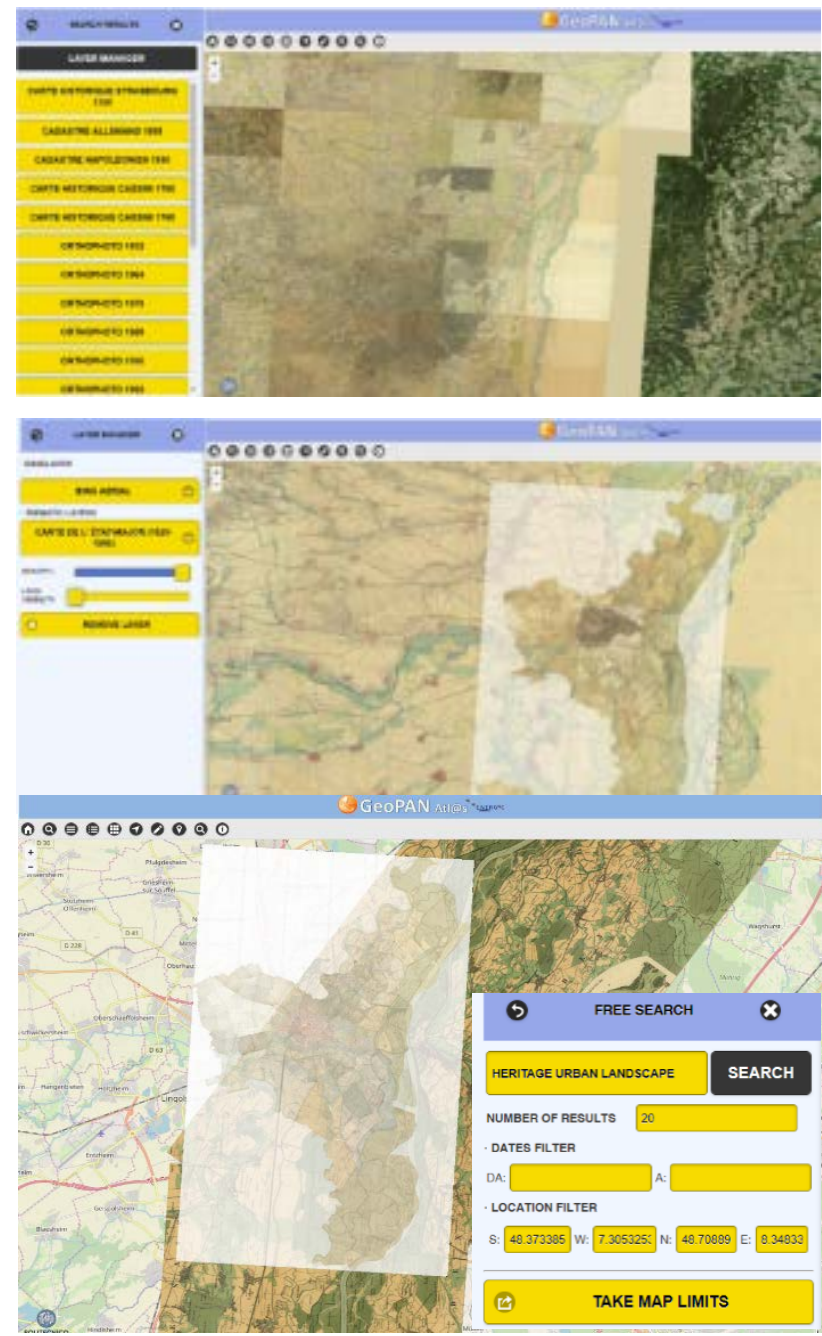

Figure 6. The GEOPAN APP access to the Strasbourg area by urban landscape query search. The different maps that can be accessed and compared together with the current maps and the historical ones published by the City of Strasbourg.

In particular, GeoPan Atl@s allows to retrieve open available datasets either starting from a free search and a "profile search" in order to make available the definition of a user profile (e.g. urban landscape planner, or bikers, etc.) for which it would be possible to identify a set of semantic keywords. Once a profile is associated to a set of predefined keywords it will be used by the different subjects belonging to the same profile for the search of datasets connected to the keywords. In the case of the case study presented in this paper the Strasbourg project can be compared together with analogous case studies, cities geoportal, regional geo-portals, design project taking in account the HUL preservation: thus allowing the citizens in the future to discover and access the different multi-temporal city maps selecting a City, or a Region or a Province. The APP, in fact, 
allows to add also current maps, as itineraries maps properly published to exploit the fruition of the built environment along the riverbeds across the centuries, or design projects.

From a technical perspective, GeoPan Atl@s is built on the basis of the well-known HTML/CSS/JS structure. This way GeoPan Atl@s can be accessed by all browsers supporting HTML5 (e.g., Safari iOS, Google Chrome, Firefox, Internet Explorer, Opera) both from desktop and mobile (e.g. tablets and smartphones). In addition, the solution is also released as an apk file (which is the package format used by Android for distribution and installation of mobile Apps). Different data formats can be managed (i.e. GEOTIFF, JSON). The GEOPAN APP access to the Strasbourg area by urban query search is illustrated in the Figure 6. Furthermore, the APP allows functionalities of digitalisation by the users, on site tracker, and export of the entities generated (Point, Line, Polygon) within common open source or commercial GIS (as QGIS, ARCGIS ESRI (C).

\section{CITY OF TOMORROW (INCLUSIVE, ECOLOGICAL, RESPECTFUL OF THE PAST, CREATIVE) \& MULTI-TEMPORAL MAP}

Urban planning, heritage preservation and its development is a dynamic process that will require in the future to boost digitised tools for use by all stakeholders. It is our aim to contribute in making Strasbourg into a leading virtual hub with multitemporal data access through the past into the future being to support planning and decision-making for interventions to ensure that the city of tomorrow is for all, drawing its strength and ambition from the active participation of its inhabitantswhich can come only through awareness and democratic access to knowledge.

The construction of the city of tomorrow, inclusive, ecological, respectful of the past, and creative, can be supported by multitemporal map systematic use not only during the analysis phase to support sustainable plans and interventions, but also after the interventions. Tools capable to improve comprehension by the citizens of the landscape characters coming from the past can help in promoting a better informed society, by communicating contents.

\subsection{The design integration within the map heritage hub: potentials toward awareness raising of citizens}

Making available at a Pan-European dimension the case study described here, with the multi-temporal heritage maps, the analysis and the design proposal in the challenging subject of 'Heritage and Development' strategy, can contribute to creating the opportunity of a common area continuously accessible by different actors thus allowing to improve preservation actions, raising awareness among planners and citizens. An hub where the different communities can meet (designer, planners, citizens, inhabitants) leveraging day by day the re-discovering of the densely stratified traces of a rich past.

Conservation of the built heritage is progressively linked to the preservation, to the maintenance of building and traditions, as in the case of the brick block masonry building of the past, defining preventive conservation as a necessity in everyday practice. In this perspective, it is necessary to have an instrument that supports the collection, analysis, comparison, archiving, sharing and management of all the data available concerning the geometry, topology, materials, structure and state of conservation of the built heritage. Such data sets can include not only the products of surveys, drawings, thematic, intangible and historical contents, but also information about maintenance or restoration activities and many other relevant forms of information (such as sketches, images, 3D city models). Videos on the urban mobility plan, notably of the tramway extension into Kehl in Germany from Strasbourg cutting through the Deux Rives area, can be linked to the maps (Figure 7).

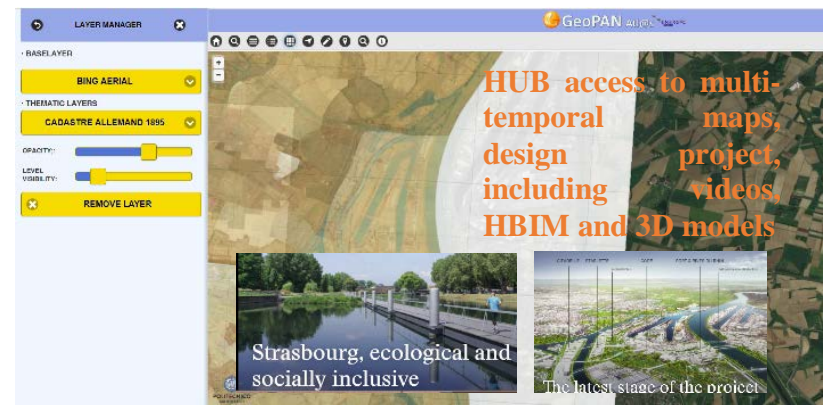

Figure 7. The integration of the available maps data with the design purposes joining landscape preservation matters.

In addition, the hub platform can now be enriched also by linked 3D model and different formats, including BIM/HBIM/IFC format (Brumana et al., 2018b) in support of HUL framework. Further recording using different technologies of surveying, as UAV orthoimages, can be implemented within the GEOPAN APP, as with the inclusion of the plans and concept designs made by different authors, agency and professionals as well as the architectural design of buildings being developed by various property developers. However, feeding with different contents the VH also implies to face IPR and copyrights guaranteeing the authorship safeguard and publicity to the contributors.

The added value represented by enriching the available data with the Virtual Reality potentials, navigation tools of the changing spaces coming from the design results and interventions can help in improving the capability of the people, habitants and tourists, to perceive the landscape values together with the design effort, without losing the important link to the past, often missed by people.

Growing consciousness by citizens, in respect to the efforts made in the Heritage and Development strategy by the HUL approach will forge the potentials of sustainable interventions, maximising heritage landscape preservation and identity, thus limiting critical impacts strengthening a circular society.

Virtual Hub capabilities toward further integration of the 3D models and HBIM design data are already available, while Virtual Reality and Augmented Reality potentials can further improve immersive comprehension of the past, together with the designed interventions. Immersive tour among different itineraries can be implemented on the hub platforms: virtual museums can be developed on the wealth of the built environment, through immersive experience via multi-temporal geodata hub (Brumana et al., 2018c).

Let's image walking or biking across the city, navigating the past and the future, with HoloLens or smartphone supported by $5 \mathrm{G}$ technology. 


\section{REMARKS}

Growing interest in urban identity, character and the historic urban landscape approach by city makers, including real estate developers, professionals and PA bodies, together with the inhabitants can be supported by technical innovative instrument capable to respond to the increased demand for historic documents of cities in constant evolution. The role of historical maps and documentation in the Heritage \& Development strategy, in respect of Heritage Urban Landscape, as recognised in the case study of the Deux-Rives Project, represents a powerful instrument to strengthening preservation aims, by maintaining the memory of the past, starting from the landscape characters to the brick block masonry adopted as a cultural identity factors. The road ahead is nonetheless full of critical concerns and necessary compromises. Innovative tools as Virtual Hubs supporting data intermediation and APPs finalized to multi-temporal navigation across past and present raising awareness by citizens in their daily life can contributes to mitigating irreversible errors and in enhancing attention to the conservation and valorisation of heritage which as nonrenewable assets, must be cherished in the sustainable development process.

\section{ACKNOWLEDGEMENTS}

The authors would like to thank the City and Eurometropole of Strasbourg, in particular, Eric Chenderowsky, Director of the Direction Urbanisme et Territoires, and Olivier Banaszak, Chief of the Service Géomatique et Connaissance du Territoire.

The research leading to the results of this paper is partially funded under the ICT Policy Support Programme (ICT PSP) of the Competitiveness and Innovation Framework Programme by the European Community (CIP) GA n620400.

\section{REFERENCES}

Brumana, R., Oreni, D., Cuca, B., Rampini, A., Pepe, M., 2013. Open access to historical information for landscape analysis in an SDI framework. International Journal of Agricultural and Environmental Information Systems (IJAEIS), 4(3), 18-40.

Brumana, R., Santana Quintero, M., Barazzetti, L., Previtali, M., Banfi, F., Oreni, D., Roels,, D., Roncoroni, F., 2015. Towards a virtual hub approach for landscape assessment and multimedia ecomuseum using multitemporal-maps. International Archives of the Photogrammetry, Remote Sensing and Spatial Information Sciences, 40, 49-53.

Brumana, R., Oreni, D., Caspani, S., Previtali, M., 2018a. Virtual museums and built environment: narratives and immersive experience via multi-temporal geodata hub. Virtual Archaeology Review, 9(19), 34-49.

Brumana R., Condoleo P., Grimoldi A., Landi A.G, Attico d., T., Banfi F. and Previtali M., 2018b. HBIM feeding OpenAccess vault inventory through GeoDB HUB, 11196 LNCS, Eds. Fink E., Brumana R.,Wallace M.,Doulamis A., Patias P., Ioannides M., Martins J., Springer Verlag, 10.1007/978-3-030-01762-0_3, pp. 27-38.

Cuca, B., Brumana, R., Oreni, D., 2013. Geo-portals: more sustainable governance of territory within spatial data framework. In First International Conference on Remote Sensing and Geoinformation of the Environment (RSCy2013) (Vol. 8795, p. 87950G). International Society for Optics and Photonics.
Mazzetti, P., Latre, M. Á., Ernst, J., Brumana, R., Brauman, S., \& Nativi, S., 2015. Virtual hubs for facilitating access to open data. In EGU General Assembly Conference (Vol. 17).

Nativi, S., Craglia, M., Pearlman, J., 2013. Earth science infrastructures interoperability: the brokering approach. IEEE Journal of Selected Topics in Applied Earth Observations and Remote Sensing, 6(3), 1118-1129.

Previtali, M. (2017). Geopan atl@s: A brokering based gateway to georeferenced historical maps for risk analysis. International archives of the photogrammetry, remote sensing and spatial information sciences, 42(2W5), 583-589.

Previtali, M., and Latre, M.Á., (2018). A brokered Virtual Hub approach for the generation of web applications based on historical maps, Appl Geomat (2018) 10: 453. https://doi.org/10.1007/s12518-018-0235-1, 453-472

UNESCO, 2011. Culture, Urban Future: Global Report on Culture for Sustainable Urban Development, Document code: CLT-2016/WS/18, http://unescodoc.unesco.org

Yang M., 2014. Agenda 21 for Culture: some reflections on the role of Cities \& Local Governments in turning principles into practice through partnerships, http://www.agenda21culture.net/sites/default/files/files/docume nts/en/newa21c_minja_yang_eng.pdf

WHITRAP, World Heritage Institute of Training and Research for the Asia and Pacific Region under the auspices of UNESCO, 2016. The HUL Guidebook: Managing heritage in dynamic and constantly changing urban environment, A practical guide to the UNESCO Recommendation on the Historic Urban Landscape, https://unesdoc.unesco.org

http://geoserver.atlas.polimi.it/examples/servlets/Geopan/D/Entr y/selector2b.html

www.strasbourgdeuxrives.eu/en/

www.sig.strasbourg.eu/2018_ImageCompare/index.html.

www.youtu.be/wroXhZ9g4sU 